\title{
The relationship between parent and child dysfunctional beliefs about sleep and child sleep
}

Article

Accepted Version

Ng, A. S., Dodd, H. F., Gamble, A. L. and Hudson, J. L. (2013)

The relationship between parent and child dysfunctional beliefs about sleep and child sleep. Journal of Child and Family Studies, 22 (6). pp. 827-835. ISSN 1573-2843 doi: https://doi.org/10.1007/s10826-012-9637-6 Available at https://centaur.reading.ac.uk/33966/

It is advisable to refer to the publisher's version if you intend to cite from the work. See Guidance on citing.

Published version at: http://link.springer.com/article/10.1007/s10826-012-9637-6

To link to this article DOI: http://dx.doi.org/10.1007/s10826-012-9637-6

Publisher: Springer

Publisher statement: This is the author's version of the manuscript. The final publication is available at http://link.springer.com/article/10.1007/s10826-0129637-6

All outputs in CentAUR are protected by Intellectual Property Rights law, including copyright law. Copyright and IPR is retained by the creators or other copyright holders. Terms and conditions for use of this material are defined in the End User Agreement. 


\section{CentAUR}

Central Archive at the University of Reading

Reading's research outputs online 
Ng, A., Dodd, H.F., Gamble, A.L., Hudson, J.L. (2013). The relationship between parent and child dysfunctional beliefs about sleep and child sleep. Journal of Child and Family Studies, 22, 827-835. doi: $10.1007 / s 10826-012-9637-6$

This is the author's version of the manuscript. The final publication is available at http://link.springer.com/article/10.1007/s10826-012-9637-6

Corresponding author: Jennifer L Hudson. Email: jennie.hudson@mq.edu.au 


\begin{abstract}
Cognitive theories emphasise the role of dysfunctional beliefs about sleep in the development and maintenance of sleep-related problems (SRPs). The present research examines how parents' dysfunctional beliefs about children's sleep and child dysfunctional beliefs about sleep are related to each other and to children's subjective and objective sleep. Participants were 45 children aged 11 -12 years and their parents. Self-report measures of dysfunctional beliefs about sleep and child sleep were completed by children, mothers and fathers. Objective measures of child sleep were taken using actigraphy. The results showed that child dysfunctional beliefs about sleep were correlated with father $(r=.43, p<.05)$ and mother $(r=.43, p<.05)$ reported child SRPs, and with Sleep Onset Latency $(r=.34, p<.05)$. Maternal dysfunctional beliefs about child sleep were related to child SRPs as reported by mothers $(r=.44, p<.05)$, and to child dysfunctional beliefs about sleep $(r=.37, p<.05)$. Some initial evidence was found for a mediation pathway in which child dyfunctional beliefs mediate the relationship between parent dysfunctional beliefs and child sleep. The results support the cognitive model of SRPs and contribute to the literature by providing the first evidence of familial aggregation of dysfunctional beliefs about sleep.
\end{abstract}




\section{Introduction}

Children frequently present to pediatric clinics with sleep related problems (SRPs), including insomnia, difficulty sleeping alone and parasomnias, such as nightmares, sleep-walking and teeth-grinding disturbances (Keren, Feldman, \& Tyano, 2001). Poor sleep in children has been linked to subsequent difficulties including conduct problems, hyperactivity, aggression, anxiety and depression (Gregory, Eley, O'Connor, \& Plomin, 2004). Considering the prevalence and consequences of child SRPs, a better understanding of the factors that underpin these difficulties is essential to determine which areas to target in prevention and treatment. In the present research, we examine the role of dysfunctional beliefs about sleep in children's SRPs, focusing specifically on the familial aggregation of dysfunctional beliefs.

In the cognitive model of insomnia, Harvey (2002) proposed that dysfunctional beliefs about sleep (DBAS) play a critical role in SRPs by maintaining the cycle of poor sleep. DBAS is a term used to refer to maladaptive perceptions and ideas about sleep that are unhelpful and increase the probability of SRPs. The pioneering scale measuring DBAS (Morin, Stone, Trinkle, Mercer \& Remsberg, 1993) assessed five belief themes: consequences of insomnia, control and predictability of sleep, sleep requirement expectations, causal attributions of insomnia and sleep-related practices. Compared to non-insomniacs, adults with chronic insomnia endorsed more dysfunctional beliefs about sleep, expressed more negativity about the consequences of insomnia, expressed more fear about losing control of their sleep and more feelings of helplessness around the predictability of sleep (Morin et al., 1993). These findings have been replicated subsequently in adult populations using various shortened versions of the original scale (e.g. Morin, Vallieres, \& Ivers, 2007).In addition to these cross-sectional findings linking dysfunctional beliefs and SRPs, treatment research with adults has shown that changes in dysfunctional beliefs are correlated with improved sleep efficiency and better maintenance of improvements at follow-up (Morin, Blais, \& Savard, 2002), suggesting that dysfunctional beliefs about sleep are not simply a correlate of SRPs but play a causal or maintaining role in SRPs.

Although some recent research has examined other dysfunctional cognitive processes associated with child sleep, such as catastrophizing (Gregory, Noone, Eley, Harvey, \& The Steps Team, 2010), only one study has examined the role of dysfunctional beliefs about sleep in child SRPs. Using a community sample of children aged 8-10 years, Gregory, Cox, Crawford, Holland and Harvey (2009) showed that child DBAS 
correlated with self and parent-reported sleep difficulties. Here we aim to extend this previous work in several ways. First, as stated by Gregory and colleagues, the DBAS-Child scale piloted in the study requires further refinement and validation. Second, the study did not include an objective physiological measure of sleep. Objective measures of sleep are an important complement to self-report and parent-report measures, which can both be affected by the reporters' own experiences and biases. For example, parents might be unaware of their child's sleep problems if the child wakes during the night and does not disturb them (Owens, Spirito, McGuinn, \& Nobile, 2000b).

Although child dysfunctional beliefs have received attention in the recent literature, as yet there has been no consideration of where these beliefs come from. Information processing theory posits that children develop schemas or 'specific rules that govern information processing and behaviour' (Beck, 1990, p.8) as part of their normal cognitive development. Children develop schemas about themselves, others and the external world. As children's schemas develop, parents may be an important source of information. For example, when asked what they know about sleep and where the information came from, children most frequently cite adults as sources of information (Owens, Stahl, Patton, Reddy, \& Crouch, 2006). There is also evidence from other areas of research, such as the child anxiety literature, that parental cognitions extend into their child's environment and can affect children's own cognitions (Lester, Field, Oliver \& Cartwright-Hatton, 2009; Lester, Seal, Nightingale \& Field, 2010; Creswell \& O'Connor, 2006.) Following this, it seems plausible that parents may act as a source of dysfunctional beliefs about sleep and that these may become integrated into the child's sleep-related schema. In particular, parent's dysfunctional beliefs about children's sleep may affect the child's own dysfunctional beliefs and ultimately their child's actual sleep. The idea that parents affect their child's sleep is not a new one and substantial research has shown that parental behaviours around sleep and bedtime are associated with child sleep (e.g. Johnson \& McMahon, 2008). However, to date there has been no consideration of how parent's dysfunctional beliefs might affect their child's sleep or their child's cognitions about sleep.

The primary goal of this research was to clarify the nature and correlates of children's dysfunctional beliefs about sleep. The first aim was to replicate and extend the finding that child dysfunctional beliefs are positively associated with child SRPs. The second aim was to investigate whether parent dysfunctional beliefs about their child's sleep are associated with child SRPs; a significant positive relationship was hypothesized in 
which more dysfunctional beliefs are associated with more SRPs. The third aim was to examine whether there is a relationship between parent and child dysfunctional beliefs; a significant positive relationship was hypothesized. In addition, the potential for child dysfunctional beliefs about sleep to mediate a relationship between parent dysfunctional beliefs about children's sleep and children's sleep problems will be examined if the initial analyses support this possibility.

\section{Method}

\section{Participants}

45 children (24 girls) aged 11 to 12 years $(M=11.2, S D=.41)$ and their mothers and fathers took part in the study. All participants were taking part in a larger longitudinal study and were initially recruited when the children were aged 4 via adverts in a free parenting magazine and through local preschools. At initial recruitment, mothers completed the Short Temperament Scale for Children (STSC; Sanson, Smart, Prior, Oberklaid, \& Pedlow, 1994) and children who scored more than 1sd above or below the normative mean on the Approach Scale were recruited. These children were classified as behaviourally inhibited $(\mathrm{N}=28)$ or uninhibited $(\mathrm{N}=17)$ respectively (see Hudson, Dodd \& Bovopoulos, 2011) for further details on the sample and recruitment). At the time that the present research was conducted, the sample mean and standard deviation on the Behavioural Inhibition Scale (Gest, 1997; Muris, Merckelbach, Wessel, van de Ven, 1999), a self-report measure of behavioural inhibition ( $\operatorname{mean}=8.78, \mathrm{sd}=3.33$ ), was consistent with community norms for this measure (mean = 9, sd = 2.6; Muris et al., 1999) and the data were normally distributed. This suggests that this sample were representative of the general population at the time they participated in this research, despite being recruited as extreme groups. Participants were predominately Australian/Oceanic (75.6\%), 15.6\% were European and $8.9 \%$ were Asian. The majority came from high income homes with $66.7 \%$ reporting an annual gross family income of over AU\$80,000.

\section{Measures}

DBAS-Child Scale (DBAS-Child). Children's dysfunctional beliefs were measured using a revised version of the DBAS-10 (Edinger \& Wohlgemuth, 2001). Adaptations were made in collaboration with the authors of the original child measure (Gregory et al., 2009) and Dr Sarah Blunden from the University of South Australia. First, "I need 8 hours of sleep" was changed to "I must have 9 hours sleep" to better reflect the higher average hours of sleep for children, as shown in recent studies of Australian children (Gradisar, 
Gardner, \& Dohnt, 2011; Teng, Bartle, Sadeh, \& Mindell, 2011). Second, word substitutions were made in four items to make them clearer for child participants (e.g. "After a poor night's sleep" was replaced with "If I don't get the sleep I need"). The ten items that make up the scale were all statements about sleep-related beliefs (e.g. "When I have trouble getting to sleep, I should stay in bed and try harder"), rated on a 5-point Likert scale ( 1 = strongly disagree, 5 = strongly agree). For the current sample, the internal consistency was good $(\alpha=.84)$.

Dysfunctional Beliefs About Child Sleep Scale (DBACS). Mothers' and Fathers' dysfunctional beliefs about children's sleep were measured using a new instrument adapted from the original DBAS scale for adults by Morin et al. (1993). The original 28 items were modified to tap into parent's dysfunctional beliefs about children's sleep. First, 6 items from the original version were excluded as they were not relevant for children (e.g. because I am getting older, I need less sleep; in order to be alert and function well during the day, I am better off taking a sleeping pill rather than have a poor night's sleep). Second, word substitutions were made to the remaining 22 items (e.g. "I have" was replaced with "my child has", "irritable, depressed, or anxious" was replaced with "annoyed, sad, or worried"). Third, two items were rephrased to not be contingent upon having SRPs (e.g. 'I believe insomnia is ruining my ability to enjoy life and prevents me from doing what I want' was changed to 'I believe insomnia could ruin my child's ability to enjoy life and prevent him/her from doing what he/she wants'). All items were stated as beliefs around the sleep of the respondent's child (e.g. "I am worried that my child may lose control over his/her sleep"). A 5-point Likert scale (1 = strongly disagree, $5=$ strongly agree) was used for consistency with the DBAS-Child scale. One item was reverse-scored. Responses were summed to give a total score. For the current sample the internal consistency of the DBACS scale was adequate for fathers $(\alpha=.66)$ and good for mothers $(\alpha=.86)$. A copy of the final items is shown in the appendix.

Child Sleep Habits Questionaire (CSHQ). The CSHQ (Owens, Spirito, \& McGuinn, 2000a) was used to measure parent-reported child SRPs. Mothers and fathers were asked to report on their child's sleep behaviours in a typical week. The CSHQ consists of 45 items forming 8 subscales which assess key sleep domains: bedtime resistance, sleep-onset delay, sleep duration, sleep anxiety, night wakings, parasomnias, sleep-disordered breathing and daytime sleepiness. Respondents were asked to rate each item on a 3-point Likert scale ( 1 = rarely: 0 to once a week, 2 = sometimes: 2 to 4 times a week, 3 = usually: 5 to 7 times a week) and to indicate whether they felt it was a problem for their child. The scale includes 10 items that elicit clinical 
information and are not included in the total score. Of the remaining 35 items, two are found in two subscales, thus 33 items were summed to give a total score.

The CSHQ has adequate test-retest reliability (range .62 to .79) and predictive validity in differentiating community vs. sleep-disordered groups (Owens et al., 2000a). Acceptable internal consistency for the 33 items has been demonstrated in both community $(\alpha=.68)$ and clinical samples $(\alpha=.78)$ (Owens et al., 2000a). For the current sample, internal consistency was adequate for the CSHQ for father-report $(\alpha=.68)$ and good for mother-report $(\alpha=.81)$.

Sleep Self Report (SSR). The SSR (Owens et al., 2000b) was used to measure children's self-reported SRPs. This 26 -item scale was designed for children aged 7 to 12 years with items approximating those in the CSHQ. Participants used a 3-point Likert scale ( 1 = rarely: 0 to once a week, 2 = sometimes: 2 to 4 times a week, 3 = usually: 5 to 7 times a week) to rate the frequency of a range of SRPs. Scores are summed to give a total score. The SSR has been shown to have good internal consistency ( $\alpha=.88$ ) (Owens et al., 2000b) and has been used in many studies on child sleep (e.g. Gregory \& Eley, 2005). For the current sample, internal consistency was adequate $(\alpha=.68)$.

Actigraphy and Sleep Diary. As an objective measure of sleep, children were asked to wear an actiwatch on their non-dominant wrist for seven nights. Actigraphs are mini motion loggers that record when the wearer is active versus inactive. Periods of inactivity are later coded as rest/sleep. Actiwatch-64 was used with recording epochs set to 1 minute. Data were analysed using Actiware-CT 5.1 (Mini Mitter, Respironics Inc., USA). This device and software have shown good concordance against polysomnography in preadolescent children (Ward, Lentz, Kieckhefer, \& Landis, 2011). Actigraphic sleep variables used in the current study included the following: Sleep Onset Latency (minutes between reported bedtime and sleep onset, defined as the first of 5 consecutive minutes of inactivity as identified by Actiware-CT 5.1 software); Total Sleep Time (minutes of sleep time excluding all periods of wakefulness); and Sleep Efficiency \% (proportion of reported duration in bed coded as sleep).

To supplement actigraphic data, participants recorded the following sleep parameters in a Sleep Diary adapted from previous research (Paine \& Gradisar, 2011): the time they went to bed; lights-out time; time spent asleep and the time they got out of bed. Sleep Diary data were used to define the period of time in bed on the actigraph and to validate the participants had used the actiwatch correctly. 
The combination of actigraphy supplemented by Sleep Diary logs is regarded as a reliable objective measure of sleep and has been used in a number of studies child sleep studies (e.g. Sadeh, Raviv, \& Gruber, 2000). This combined method has the advantage of external validity by measuring sleep in a naturalistic setting.

\section{Procedure}

The procedures were approved by the University Ethics Committee. Parents provided written consent for themselves and their child to participate. Initial information and consent forms were posted to parents who were then contacted by phone and invited to take part in the study. Upon consent, parents were sent links to online questionnaires, a paper copy of the Sleep Diary for their child to complete daily and programmed actiwatch with instructions. During the week of data collection, an SMS was sent each evening to remind participants to use their actiwatch and Sleep Diary. Participants returned their actiwatch and Sleep Diary after 7 nights of data collection. Participants were entered into a draw for one of two iPod shuffles.

\section{Data Preparation}

Variables were exported to a report and all data were saved to the Actiware database. Due to children and parents choosing not to complete online questionnaires, 7 participants had missing data for the SSR, 6 for the DBAS-Child, 10 for the father-report DBACS and CSHQ, and 5 for the mother-report DBACS and CSHQ. Research has shown that actigraphy is reliable when five or more nights of data is available (Acebo et al., 1999). Consequently, one participant was excluded for collecting less than 5 nights of data. Three other participants were excluded because of questions about the reliability of their actigraphy data: one whose Sleep Diary data was extremely discrepant from their actigraphic data indicating incorrect use of the actiwatch; one who reported that their sleep had been disrupted by the actiwatch for the first few nights; one who reported highly unusual sleeping patterns due to being on school holidays. Analyses were conducted with all available data.

All variables were checked for conformity to the assumption of normal distribution. The motherreport CSHQ was positively skewed and was corrected using a square-root transformation. The transformed variable was used for all analyses.

Demographic data were analysed and no significant effects due to sex, age, ethnicity or gross family income were found for any of the dependent variables $(p>.5)$. To examine whether the nature of the sample 
might affect the results, independent samples t-tests were conducted to examine the relationship between $\mathrm{BI}$, parent and child dysfunctional beliefs and child sleep. No significant differences were found between the BI and uninhibited groups on any of the measures. Given this finding, BI was not controlled for in any analyses.

Given the relatively small sample size and the exploratory nature of some of the research questions an unadjusted $p$-value of $p<.05$ was used as the criteria for statistical significance. This is consistent with the presentation of findings in past research exploring dysfunctional beliefs in adults (Morin et al., 1993) and children (Gregory et al., 2009).

\section{Results}

Pearson's correlations were conducted to examine relationships between child dysfunctional beliefs about sleep, parent dysfunctional beliefs about child sleep, and measures of child sleep. The results are shown in Table 1.

\section{The relationship between measures of child sleep}

There was some evidence that the questionnaire measures of child sleep were related, with significant correlations between mother-report of child sleep problems on the CSHQ and father and child report of child sleep problems. Similarly, there was some evience the actigraphy variables were related, with Sleep Efficiency significantly correlated with Sleep Onset Latency and Total Sleep Time (note that this is expected given that Sleep Efficiency is calculated based on Sleep Onset Latency and Total Sleep Time). No signifcant correlations were found between actigraphy and self-report variables. Non-significant correlations between the measures of child sleep may be due to the different measures assessing disperate aspects of SRPS.

[Insert Table 1 here]

\section{Child Dysfunctional Beliefs About Sleep and Child SRPs}

A significant positive correlation was found between total scores on the DBAS-Child and motherreport CSHQ $(r=.43, p=.008)$ as well as father-report CSHQ $(r=.43, p=.01)$. DBAS-Child total score showed a moderate positive correlation with Sleep Onset Latency $(r=.34, p=.04)$ but not with child-reported sleep problems, Sleep Efficiency or Total Sleep Time $(p>.05)$.

Parent Dysfunctional Beliefs About Child Sleep and Child SRPs 
There was a significant moderate correlation between scores on the mother-report DBACS and mother-report CSHQ $(r=.44, p=.005)$ but not any of the other sleep variables. Correlations between fatherreport DBACS scores and the measures of child sleep did not reach significance.

\section{Child Dysfunctional Beliefs about Sleep and Parent Dysfunctional Beliefs About Child Sleep}

A significant positive correlation was found between DBAS-Child and mother-report DBACS scores $(r=$ $.37, p=.02$ ). Additionally, father and mother-report DBACS scores were also positively correlated with each other $(r=.348, p=.04)$. No significant correlation was found between father-report DBACS and DBAS-child. Child dysfunctional beliefs as a mediator of the relationship between maternal dysfunctional beliefs and child SRPs

Given that a significant relationship was found between maternal DBACS and child sleep (motherreport CSHQ), between maternal DBACS and child DBAS and between child DBAS and child sleep (motherreport (SHQ), it is plausible that child DBAS may mediate the relationship between maternal DBACS and child sleep. The above three significant correlations meet the first three criteria specified by Baron and Kenny (1986) in their definition of mediation. The fourth criterion, that the direct effect of maternal DBACS on child sleep becomes nonsignificant after controlling for the effect of the potential mediator (child DBAS) can be assessed using simple regression. However, this approach has been criticised in recent papers (e.g. Preacher \& Hayes, 2004) and a more thorouogh investigation of mediation including an estimation of the indirect effect is generally recommended. Using the SPSS indirect macro described in Preacher and Hayes (2004), which provides a bootstrapped estimation of the indirect effect and $95 \%$ confidence intervals the following results were found. The three relationships desribed above were all statistically significant, as anticipated. The direct effect of maternal DBACS on Child sleep after controlling for Child DBAS was not significant $($ coeff $=.02$, se $=$ $.01, \mathrm{t}=1.28, p=.21)$, indicating complete mediation as defined by Baron and Kenny (1986). In addition the 95\% confidence intervals around the bootstrapped estimates of the indirect effect did not include zero (lower $=.0015 ;$ upper $=.0278)$ indicating a significant indirect effect from maternal DBACS to Child Sleep via Child DBAS. 


\section{Discussion}

The aim of this research was to examine the nature and correlates of dysfunctional beliefs about sleep in children. There were three overarching hypotheses: 1 . that children's dysfunctional beliefs about sleep would be associated with child SRPs; 2 . that parent dysfunctional beliefs about children's sleep would be associated with child SRPs; 3 . that parent and child sleep-related cognitions would be associated. The results provided some support for these hypotheses.

As anticipated based on previous research (Gregory et al., 2009; Morin et al., 1993), there was some evidence that child dysfunctional beliefs about sleep were associated with child SRPs. Child dysfunctional beliefs were positively correlated with mother and father report of child SRPs. Furthermore, although it was not significant, there was a moderate correlation between child DBAS and child-reported sleep problems on the SSR. This correlation is comparable to that reported by Gregory et al. (2009) but a larger sample size would be required for a correlation coefficient of this magnitude to reach statistical significance (Taylor, 1990) , thus the results somewhat replicate the findings of Gregory and colleagues. In addition to questionnaire measures, child dysfunctional beliefs were also associated with Sleep Onset Latency, although not with Total Sleep Time or Sleep Efficiency. The specific relationship found between child dysfunctional beliefs about sleep and longer Sleep Onset Latency replicates findings in the adult literature (Wicklow \& Espie, 2000) and may be due to children with these cognitions being worried about getting enough sleep and feeling pressured to fall asleep quickly, which may specifically affect time to fall asleep (Watts, Coyle, \& East, 1994). This interpretation is consistent with the cognitive model of insomnia, which proposes that dysfunctional beliefs are associated with negative sleep-related thoughts at the time of going to bed, which lead to increase cognitive arousal and difficulty falling asleep (Harvey, 2000).

With regards the relationship between parent dysfunctional beliefs about children's sleep and child SRPs, mothers' dysfunctional beliefs were found to be related to child SRPs, but only as reported by mothers (no relationship was found with child-reported SRPs or objective measures of child sleep). There was no evidence that fathers' dysfunctional beliefs were related to child SRPs. The significant relationship found for mothers must be treated with caution due to the shared method variance between these measures. However, the finding is in line with the association between dysfunctional maternal cognitions about bedtime care and infant SRPs reported in the infant sleep literature (Johnson \& McMahon, 2008). It is plausible that mothers 
continue to convey their sleep-related cognitions to their children beyond infancy, and these beliefs continue to be related to child SRPs in late childhood. It is also possible, of course, that children's SRPs affect their mother's cognitions.

The results suggest that there may be a relationship between child and mother cognitions, with children who have mothers with dysfunctional beliefs about children's sleep more likely to have dysfunctional beliefs themselves. In contrast, there was little evidence for an association between child and father cognitions. Taken together with the findings outlined above, the results suggest that father cognitions are not closely related to child cognitions or child sleep. There may be several possible explanations for this pattern. It is plausible that fathers have less involvement around children's bedtime, perhaps as an artifact of nursing responsibilities from infancy, or stereotypical gender roles within families (Australian Bureau of Statistics, 2007; Tikotzky, Sadeh, \& Glickam-Gavrieli, 2011). Alternatively, fathers may be less vocal about their beliefs around sleep and hence their beliefs are less likely to affect the child's sleep and sleep-related beliefs. It should also be acknowledged that the internal consistency for the father-report DBACS and CSHQ scales was modest, hence the findings from paternal data require some caution in interpretation. Nevertheless, the inclusion of fathers' cognitions remains important as the role of fathers has been neglected in this area and, by studying both parents, we can gain insight into the relative importance of mothers and fathers. It is also important to note that the dysfunctional beliefs about children's sleep measure, completed by parents, was adapted from the original DBAS measure (Morin et al., 1993) which has a different factor structure to the ten-item measure from which the DBAS-Child was adapted (Edinger \& Wohlgemuth, 2001). These measures are therefore be assessing slightly different dysfunctional beliefs about sleep, which might also explain the relatively low correlations between parent and child cognitions. It is noteworthy that there was a moderate association between fathers' and mothers' dysfunctional beliefs about children's sleep. This seems fairly intuitive, as parents are likely to discuss their thoughts about their approach to child sleep with each other and develop shared attitudes. This additional finding supports the idea that there is a familial component to dysfunctional beliefs about sleep.

As discussed, it is plausible that parents' dysfunctional beliefs about their child's sleep affect their child's own dyfunctional beliefs, which in turn affect the child's sleep. That is, that child DBAS mediate the relationship between parent DBACS and child sleep. Based on the initial results already discussed and the 
criteria for mediation outlined by Baron \& Kenny (1986), only child sleep as measured by mother-report using the Child Sleep Habits Questionnaire and maternal DBACS were eligible for consideration in regards to this hypothesis. The results provided some initial support for a mediation pathway, with child DBAS completely mediating the relationship between maternal DBACS and mother-report of child sleep problems. This result provides some initial suggestion that mother's might affect their child's sleep via the transfer of their own dysfunctional beliefs about sleep. It is important to bear in mind, however, that the data are cross-sectional so some caution is required in interpreting the findings. In addition, the mediation pathway was only found for mother DBACS and only found for maternal report of child sleep problems. Further investigation of this pathway will be useful in larger samples and may have important implications for the involvement of parents in treatment for child sleep problems.

The present research has a number of strengths and contributes to the literature in a number of ways. This is the first study to have assessed parent's dysfunctional beliefs about children's sleep. The results show that parents hold beliefs about children's sleep and that mothers' beliefs may affect the child's own beliefs and, at the very least, their perception of their child's sleep. The inclusion of both mothers and fathers is an important strength and provides some interesting insights into how the relationship between parent beliefs, child beliefs and child sleep differs between mothers and fathers. Another strength of the present research is the use of actigraphy as a measure of child sleep. This is especially advantageous when studying the relationship between dysfunctional beliefs and sleep, as children who have more dysfunctional beliefs would perhaps be more inclined to overestimate their SRPs. Finally, this study has provided further testing and validation of the DBAS-child scale (Gregory et al., 2009) and has developed and piloted a new scale that measures parent's dysfunctional beliefs about children's sleep (DBACS). This new scale complements the scale developed by Morrell (1999), which assesses maternal cognitions about infant sleep, in a number of ways. First, the new scale focuses specifically on dysfunctional beliefs about children's sleep, whereas the Morrell scale includes a wider range of cognitions on themes such as doubting ones parenting ability (e.g. If I say no to my child's demands at night, then that means I'm a bad mother') and more practical issues such as safety during the night and feeding (e.g. when my child wakes during the night, I think I might not have fed him/her enough during the day). Second, the new measure has been developed for use with parents of older children, rather than infants, and the items are designed to be appropriate for a wide age range. 
An important caveat to the present study is the cross-sectional nature of the research. It is important to bear in mind that none of the current findings provide insight into the causal status of the relationships studied. For instance, the current data suggest some association between child dysfunctional beliefs about sleep and SRPs but the direction of this relationship remains ambiguous; children's dysfunctional beliefs may lead to poor sleep but it is equally likely that poor sleep created the dysfunctional beliefs in the first place, or that a bidirectional relationship exists. Similarly, maternal dysfunctional beliefs about child sleep were related to child SRPs and to child dysfunctional beliefs but it is not possible to determine from the current study what causes what. Whilst it may be intuitive to think parents are the root of child cognitions and behaviour, the opposite relationship is also possible; a child who expresses poor sleep and dysfunctional beliefs about sleep may cultivate maternal dysfunctional beliefs. One way of investigating causality in future studies would be to conduct longitudinal research to assess how parent dysfunctional cognitions, child dysfunctional cognitions and child SRPs affect each other over time. As a complement to this type of research, future studies could also target maternal and child dysfunctional beliefs about sleep in CBT-I for children. Previous studies have demonstrated the efficacy of CBT-I in modifying dysfunctional beliefs about sleep in adults (Edinger, Wohlgemuth, Radtke, Marsh, \& Quillian, 2001), which once corrected, lead to improved sleep efficiency and maintained improvements at follow-ups (Morin et al., 2002). Studies demonstrating the efficacy of CBT-I for children have been published recently (Paine \& Gradisar, 2011). It will be useful for future studies to examine how dysfunctional beliefs change across the course of treatment and whether changes in dysfunctional beliefs mediate changes in child sleep problems. Another area which will be of interest for future research is further examination of other dysfunctional cognitions related to child sleep such as catastropizing. Gregory et al. (2010) found an interesting relationship between catastrophizing cognitions and child sleep problems that was mediated by anxiety and depression symptoms. There remain many interesting questions in this area including how catastrophizing cognitions are related to dysfunctional beliefs and whether parents act as a source of catastrophizing cognitions.

Other limitations that should be considered include the relatively small sample size; it is possible that some of the analyses would have reached significance with a larger sample. Additionally, the participants in the current sample were selected as being inhibited or uninhibited when they were aged 4 . It is therefore possible that the current results were affected by the nature of the sample. Preliminary analyses negated this 
possibility, however, as behavioural inhibition was not associated with any of the other measures. Additional directions for future research include further refinement and validation of the DBAS-Child and DBACS scales and examination of parents' dysfunctional beliefs about their own sleep as well as about children's sleep.

Overall the current study contributes to the literature by providing the first examination of how child dysfunctional beliefs about sleep are related to objectively measured SRPs, and evaluating the role of mother and father dysfunctional beliefs in child sleep and sleep-related cognitions. The current study provides evidence that child dysfunctional beliefs about sleep are associated with child SRPs as reported by mothers and fathers, and with Sleep Onset Latency. The results support the familial aggregation of dysfunctional beliefs about sleep, although the precise mechanisms by which parent and child dysfunctional beliefs and SRPs interact remains to be addressed in future research. Understanding the role of the family and dysfunctional beliefs will ultimately inform treatment and prevention of child sleep problems.

\section{Acknowledgements}

The authors would like to thank Talia Morris for her help with data collection and support, Leigh Stanger for his technical support and the many parents and children who generously donated their time to our research. This project was supported by the Australian Research Council Discovery Grant (DP0342793).

\section{References}

Acebo, C., Sadeh, A., Seifer, R., Tzischinsky, O., Wolfson, A.R., Hafer, A., \& Carskadon, M. (1999). Estimating sleep patterns with activity monitoring in children and adolescents: how many nights are necessary for reliable measures? Sleep, 22, 95-103.

Australian Bureau of Statistics. (2007). Fathers' Work and Family Balance. Australian Social Trends, 2006.

Baron, R.M., \& Kenny, D.A. (1986). The moderator-mediator variabl distinction in social psychological research: Conceptual, strategic and statistical considerations. Journal of Personality and Social Psychology, 51, 1173-1182.

Beck, A.T., Freeman, A., Pretzer, J., Davis, D.D., Fleming, B., Ottavani, R., ...\& Trexler, L. (1990). Cognitive Therapy of Personality Disorders. New York: Guildford Press. 
Creswell, C. \& O'Connor, T. G. (2006). 'Anxious cognitions' in children: An exploration of association and mediators. British Journal of Developmental Psychology, 24, 761-766.

Edinger, J.D., \& Wohlgemuth, W.K. (2001). Psychometric comparisons of the standard and abbreviated DBAS10 versions of the dysfunctional beliefs and attitudes about sleep questionnaire. Sleep Medicine, 2, 493-500.

Edinger, J. D., Wohlgemuth, W. K., Radtke, R. A., Marsh, G. R. \& Quillian, R. E. (2001). Does cognitivebehavioral insomnia therapy alter dysfunctional beliefs about sleep? Sleep, 24, 591-599.

Gest, S. D. (1997). Behavioral inhibition: stability and associaitions with adaptation from childhood to early adulthood. Journal of Personality and Social Psychology, 72, 467 - 475.

Gradisar, M.S., Gardner, G.P., Dohnt, H.K. (2011). Recent world-wide sleep patterns and problems during adolescence: A review. Sleep Medicine, 12, 110-118.

Gregory, A. M., Cox, J., Crawford, M. R., Holland, J. \& Harvey, A. G. (2009). Dysfunctional beliefs and attitudes about sleep in children. Journal of Sleep Research, 18, 422-426.

Gregory, A. M. \& Eley, T. C. (2005). Sleep problems, anxiety and cognitive style in school-aged children. Infant and Child Development, 14, 435-444.

Gregory, A. M., Eley, T. C., O'Connor, T. G. \& Plomin, R. (2004). Etiologies of associations between childhood sleep and behavioral problems in a large twin sample. Journal of the American Academy of Child and Adolescent Psychiatry, 43, 744-751.

Gregory, A.M., Noone, D.M., Eley, T.C., Harvey, A.G., \& The Steps Team. (2010). Catastrophizing and symptoms of sleep disturbances in children. Journal of Sleep Research, 19, 175-182.

Harvey, A. G. (2002). A cognitive model of insomnia. Behaviour Research and Therapy, 40, 869-893.

Hudson, J.L., Dodd, H.F. \& Bovopoulos, N. (2011). Temperament, family environment and anxiety in preschool children. Journal of Abmormal Child Psychology, 39, 939-951.

Johnson, N. \& McMahon, C. (2008). Preschoolers' sleep behaviour: Associations with parental hardiness, sleeprelated cognitions and bedtime interactions. Journal of Child Psychology and Psychiatry, 49, 765-773.

Keren, M., Feldman, R. \& Tyano, S. (2001). Diagnoses and interactive patterns of infants referred to a community-based infant mental health clinic. Journal of the American Academy of Child and Adolescent Psychiatry, 40, 27-35. 
Lester, K. J., Field, A. P., Oliver, S., \& Cartwright-Hatton, S. (2009). Do anxious parent's interpretive biases toward threat extend to their children's environment? Behaviour Research and Therapy, 47, 170-174.

Lester, K. J., Seal, K., Nightingale, Z. C., \& Field, A. P. (2010). Are children's own interpreations of ambiguous situations based on how they perceive their mothers have interpreted ambiguous situations for them in the past? Journal of Anxiety Disorders, 24, 102-108.

Morin, C. M., Blais, F. \& Savard, J. (2002). Are changes in beliefs and attitudes about sleep related to sleep improvements in the treatment of insomnia? Behaviour Research and Therapy, 40, 741-752.

Morin, C. M., Stone, J., Trinkle, D., Mercer, J. \& Remsberg, S. (1993). Dysfunctional beliefs and attitudes about sleep amongst older adults with and without insomnia complaints. Psychology and Aging, 8, 463-467.

Morin, C.M., Vallieres, A., \& Ivers, H. (2007). Dysfunctional beliefs and attitudes about sleep (DBAS): Validation of a brief version (DBAS-16). Sleep, 30, 1547-1554.

Morrell, J.M.B. (1999). The Role of Maternal Cognitions in Infant Sleep Problems as Assessed by a New Instrument, the Maternal Cognitions about Infant Sleep Questionnaire. Journal of Child Psychology and Psychiatry, 40, 247-258.

Muris, P., Merckelbach, H., Wessel, I., van de Ven, M. (1999). Psychopathological correlates of self-reported behavioural inhibition in normal children. Behaviour Research and Therapy, 37, 575-584

Owens, J. A., Stahl, J., Patton, A., Reddy, U. \& Crouch, M. (2006). Sleep practices, attitudes and beliefs in inner city middle school children: A mixed methods study. Behavioural Sleep Medicine, 4, 114-134.

Owens, J. A., Spirito, A., \& McGuinn, M.(2000a) The Children's Sleep Habits Questionnaire (CSHQ): Psychometric properties of a survey instrument for school-aged children. Sleep, 23, 1043-1051.

Owens, J. A., Spirito, A., McGuinn, M. \& Nobile, C. (2000b). Sleep habits and sleep disturbance in elementary school-aged children. Journal of Developmental and Behavioural Pediatrics, 21, 27-34.

Paine, S. and Gradisar, M. (2011). A randomised controlled trial of cognitive-behaviour therapy for behavioural insomnia of childhood in school-aged children. Behaviour Research and Therapy, 49, 379-388.

Preacher, K.J. \& Hayes, A.F. (2004). SPSS and SAS procedures for estimating indirect effects in simple mediation models. Behavior Research Methods, Instruments and Computers, 36, 717-731.

Sadeh, A., Raviv, A. \& Gruber, R. (2000). Sleep patterns and sleep disruptions in school-age children. Developmental Psychology, 36, 291-301. 
Sanson, A., Smart, D., Prior, M., Oberklaid, F. \& Pedlow, R. (1994).The structure of temperament from age 3 to 7 Years: Age, sex, and sociodemographic influences. Merrill-Palmer Quarterly, 40, 233-252.

Taylor, R. (1990). Interpretation of the correlation coefficient: A basic review. Journal of diagnostic medical sonography, 6, 35-39.

Teng, A., Bartle, A., Sadeh, A., \& Mindell, J. (2011). Infant and toddler sleep in Australia and New Zealand.Journal of Pediatrics and Child Helath, 48 (3), 268-273.

Tikotzky, L., Sadeh, A., \& Glickman-Gavrieli, T. (2011). Infant sleep and paternal involvement in infant caregiving during the first 6 months of life. Journal of Pediatric Psychology, 36, 36-46.

Ward, T.M., Lentz, M., Kieckhefer, G.M., \& Landis, C.A. (2011). Polysomnography and actigraphy concordance in juvenile idiopathic arthritis, asthma and healthy children. Journal of Sleep Research, 21, 113-121.

Watts, F. N., Coyle, K. \& East, M. P. (1994). The contribution of worry to insomnia. British Journal of Clinical Psychology, 33, 211-220.

Wicklow, A., \& Espie, C.A. (2000). Intrusive thoughts and their relationship to actigraphic measurement of sleep: towards a cognitive model of insomnia. Behaviour Research and Therapy, 38, 679-693. 
Table 1

Pearson's correlations between child dysfunctional beliefs about sleep, parent dysfunctional beliefs about child sleep and child sleep variables

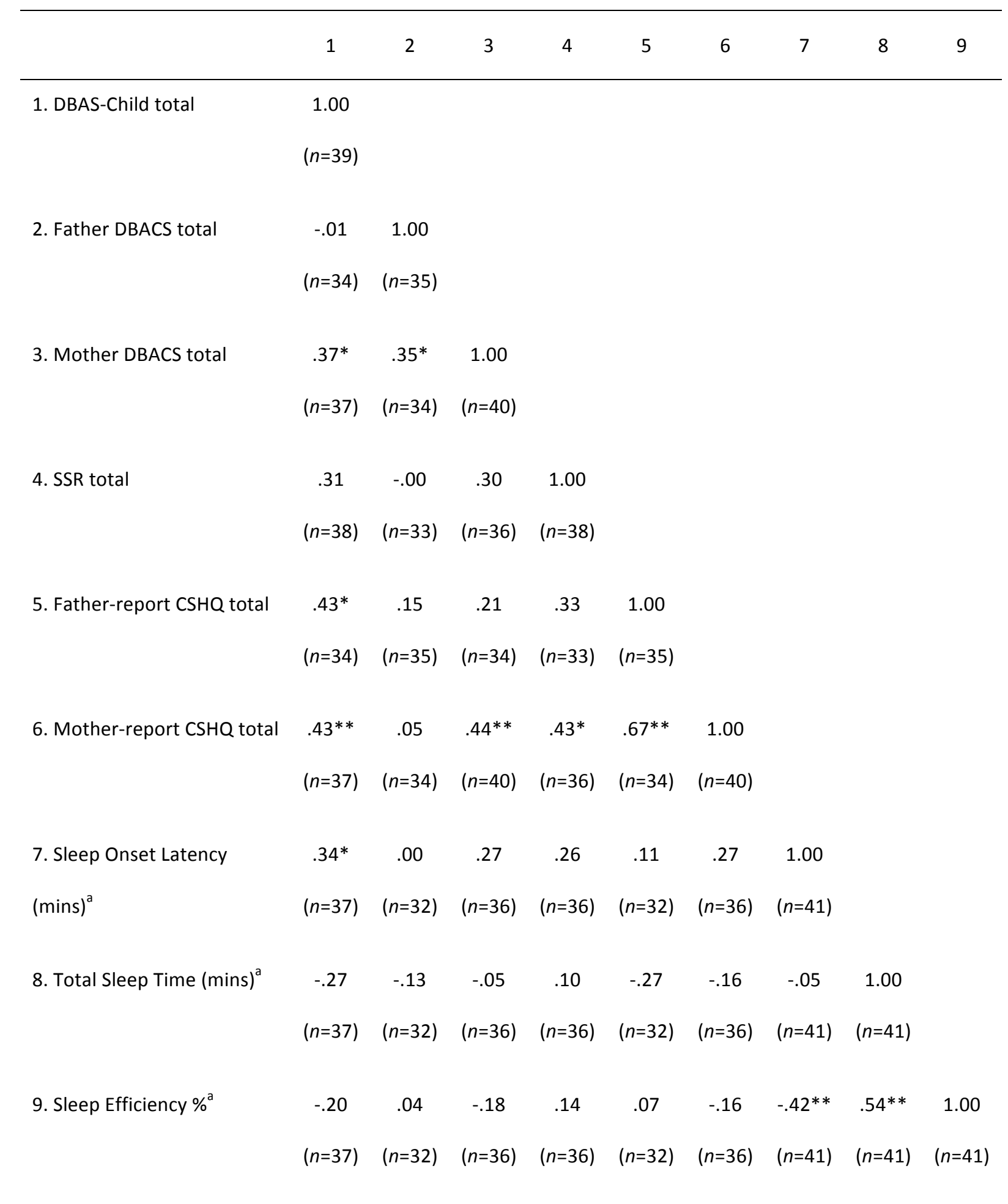


Note DBAS-Child total $=$ Dysfunctional Beliefs about Sleep child total; Father DBACS total = Father Dysfunctional Beliefs about Child Sleep total; Mother DBACS total = Mother Dysfunctional Beliefs about Child Sleep total; SSR total = Sleep Self-Report total; Father-report CSHQ total = Father-report Child Sleep Habits Questionnaire total; Mother-report CSHQ total = Mother- report Child Sleep Habits Questionnaire total. 
